\title{
Beitrag zur Kenntniss der Embolie der Arteria centralis retinae.
}

\author{
Von
}

Prof. Herm. Schmidt in Marburg.

(Hierzu Tafel III.)

Bisher sind erst zwei Fälle bekannt, bei denen sich Gelegenheit fand, die während des Lebens diagnosticirte Embolie der Arter. centralis retinae auch post mortem durch die Section zu bestätigen. Es sind dies die von v. Graefe-Schweigger*) and Sichel fils**) beschriebenen.

Ich kam nunmehr eine dritte Beobachtung hinzufügen, welche auch sonst, sowohl durch den anatomischen Befund, wie durch den Krankheitsverlauf, interessant erscheint und vielleicht dazu dient, einige dunkle Punkte bei dem in Rede stehenden Process zu lichten.

Conrad Rein, 58 Jahr alt, wurde am 3. Mai 1871 wegen Schwäche der linksseitigen Extremitäten in die

*) Arch. für Ophthalmologie IV, 2, p. 230 und Schweigger, Vorlesungen ther den Gebrauch des Augenspiegels p. 140.

*⿻) Archives de physiologie, T. IV, 1871-1872, p, 83 . 
KInik meines Collegen Mannkopff - dem ich die Möglichkeit zu dieser Beobachtung verdanke - aufgenommen. Die Parese erschien als Folge embolischer Heerde in dex rechten Hirnhemisphäre, da sich bei der Untersuchung ein complicirtes vitium cordis herausstellte.

In der Nacht vom 23, zum 24, Juli bemerkte der Patient, welcher früher nie über sein Sehrermögen gellagt hatte, dass, während er auf dem Nachtgeschirr sass, sein linkes Auge plötzlich erblindete. Die am Morgen gemachte Seh Prüfung ergab vollständige Amaurose.

Als ich den Patienten am Abend zum ersten Male sah, fand ich Folgendes: Der linke Angapfel äusserlich normal, ebenso die Tension; Pupille (es war Atropin eingeträufelt wordien) von mehr als mittlerer. Weite. Die brechenden Medien klur und durchsichtig. Der Augenspiegel zeigte die papilla optica ziemlich scharf abgegrenzt, von röthlicher normaler Fürbung. Die Arterien auf ihr sind blatleer, kaum als ganz dïnne Stränge erkennbar, aber noch ausserhalb des Opticus-Eintrittes eine Strecke weit in die Netzhaut hinein zu verfolgen. Auf Druck gegen den Augapfel tritt kein Arterien-Puls auf. Die Venen präsentiren sich auf der Netzhant als dunkle, blaurothe, zienlich dicke Surche. Desonders auffallig war der Maingel der sonst vorhandenen feineren Biegungen. An einzeinen Stellen zeigte sich eine Unterbrechung in der Continuität der in ihnen befindlichen Blutsäule. Die Gegend der Macula lutea and weiter nach der Papille zu ist leicht grau getribbt; der gelbe Fleck selbst tritt nicht, wie sonst im umgelenrten Bilde, als Queroval mit braunrother, etwas stumpier Färbang hervor. An einer Stelle, die der Lage nach etwa dem unteren Rande der Macula entsprechen würde, findet sich eine dicke, dunkelrothe, quergestellte und etwa einen halben Papillen. durchmesser lange Linie. 
Das rechte Auge ist gesund.

Am folgenden Morgen wurde eine geringe Chemosis der Conjunctiva bemerkt, die im Laufe des Tages noch zunahm, so dass sie Abends 7 Uhr die Cornea wallförmig umgab. Der Bulbus selbst trat jetzt - im Verhältniss zum rechten Auge - deutlich etwas aus der Orbita hervor und war stärker gespannt. Die Lider, ebenso die nächstliegende Parthie der Wangenhaut sind leicht ödematös. Vordere Kammer und Cornea klar. Iris etwas entfärbt, das Gewebe gelockert. Pupille, trotz Atropinisirung von nicht mittlerer Weite; auf der Linsenkapsel nach aussen gelegen, ein Halbring von ziemlich curchsichtigem Exsudat, etwa dem Pupillar-Rande bei enger Pupille entsprechend. Der Glaskörper erscheint weniger durchsichtig, doch sind grössere, geformte Trübuagen nicht zu erkennen. Der Augen-Hintergrund etwas verschleiert. Die Papilla optica ist jetzt fast gar nicht gegen die Retina abgegrenzt, röthlich, trüb. Die Venen sind noch als dunkle Stränge, mit den angegebenen Onterbrechungen der Blutsäule zu erkennen. Die Arterien immer noch ausserordentlich dünn, aber vielleicht etwas deutlicher als am Tage zuvor. Die graue Trübung in der Netzhaut hat zugenommen; der dunkelrothe Fleck in der Gegend der macula lutea ist nicht mehr zu erkennen. - Am Abend hat sich das Exsudat im Pupillar. Gebiet noch vermehrt, ist aber noch ziemlich durchsichtig, Die ganze Netzhaut erscheint grauröthlich getrubt. - Am 27. Abends ungefïhr stat. idem. Chemosis conjunctivae und Oedem der Lider. Geformte Glaskörper-Trübungen sinれ noch nicht zu sehen. Der Augenhintergrund bietet fast nur das Bild einer Retinitis; keine Schwellung der Papille. - Am 1. August sind Chemosis und Exsudat in der Pupille verringert; hingegen ist der Glaskôrper so intensiv getrübt, dass der Augen-Hintergrund bei der Spiegel-Untersuchung eben nur noch röth- 
lich durchschimmert, ohne Details erkemmen zu lassen. Am 7. August immer noch Exophthalmus, mässige Chemosis und Röthung der Conjunctiva. Pupille trotz. Atropin kaum mittelweit; das Exsudat in ihr ist verschwunden. Im etwas durchsichtigeren Glaskörper sind jetzt geformte, fadenförmige Trübungen au bemerken. Die papilla optica, welche, wenn auch ziemlich verschleiert, wieder sichtbar wird, geht unabgegrenzt in die Retina über und ist vorzugsweise an dem Conflux der dicken Venenstämme erkennbar.

Von nun an verloren sich die Erscheinungen der Irido Chorioiditis allmälig immer mehr. An 26. November ist das Auge dem Aeusseren nach normal. Cornea und vordere Kammer sind frei. Pupille klein, starr, mit hinteren Synechien. Glasköpper durchsichtig; ohne grössere Trubungen. Der Augenspiegel zeigt das Bild der Atrophia papillae opticae et retinae. Der Opticus ist sowohl wegen des Gefäss-Schwundes, als auch weil anliegende Parthien, an denen die Chorioidea zu Grunde gegangen, dieselbe weisse Färbung haben, schwer zu erkennen. Zwei dünne Gefässäste treten auf ihm efwas deutlicher hervor und gehen nach aussen hin (umgekehrtes Bild) über eine grössere weisse, unregelmässig mit kleinen grauen und schwarzen Flecken gesprenkelte Parthie. Eine ähnliche weisse, aber kleinere Fläehe zeigt sich auch nach innen won der Papille, gegen den binteren Augenpol hin sich erstreckend: die Grenze bilden hier und da schwarze Pigmentsreifen. Fon dem Opticuseintritt nach unten hin geht ein sehr dünnes Gefäss, welches sich in kleinen Schlängelungen nacb der Peripherie hinzieht, wo es stärker und deutlicher wird. In der Yetzhaut finden sich hier und da kleine Apoplexien und schwarze Pigmentflecke. Die Chorioidea zeigt ebenfalls Pigment-Alterationen. Die Peripherie des Augenhintergrundes bietet im Ganzen ein weniger verändertes 
Aussehen und einen grösseren Gefässreichthum. - Die Spannung des Bulbus ist wieder ziemlich normal. Kein quantitative Licht-Empfindung.

Der Patient starb am 27. Mai 1872. Die Section ergab in der rechten Hirnhemisphäre in der Nähe der Ventrikels einen kleinen braungefärbten Heerd von über Linsengrösse. Der linke Opticus entschieden etwas dünner als der rechte. In der medulla oblongata sinuöse Erweiterung des Centralcanals, ausgesprochene Atrophie des rechten Seitenstranges. Das Herz von colossaler Grösse. Die Wand der linken Kammer stark verdickt; an den Aortenklappen Atherom. Die hintere Klappe atm rechten Rande retrahirt.

Nach Eröffnung des Orbitaldaches präparirte ich den linken Bulbus mit möglichster Schonung des Stammes der Arteria ophthalmica und ibres zum Sehnerv gehenden Astes heraus. Derselbe gab, ehe er die Richtung zum Nerven einschlug, verschiedene Verzweigungen ab, die zum Theil nach rückwärts verliefen, zum Theil die Richtung nach dem Bulbus hin einschlugen (wohl hintere Ciliaräste).

Nach Erhärtung des Präparates ergab die genauere Untersuchung Folgendes:

In der Art. ophthalmica und den Haupt-Gefässästen war das Lumen frei, nur hier und da sassen an der Gefässwand Reste geronnenen Blutes. An einem Knotenpunkte, von wo aus sich die Arteria central. retinae abzweigte, war ein stärkeres Blutgerinnsel und in einzelnen kleinen, hier entspringenden Arterien zeigte sich, gleichwie in den mit ihnen in derselben Scheide liegenden Venen, eine thrombotische Verstopfung. Auf micro. scopischen Durchschnitten war das Lumen vollkommen ausgefüllt mit dicht an einander liegenden Blutkörperchen. Das Arterienrohr der Central. retinae war bis zur Sehnervenscheide offen, wenngleich ebenfalls auf der Wand sich zuweilen Blutgerinusel fand. Der Durchtritt durch 
die Scheide erfolgte in einer Entfernung von etwa $10 \mathrm{Mm}$. vom Bulbus.

Es wurden nun von hier aus bis in die Nähe der lamina cribrosa Querschnitte durch den Opticus gelegt. Hierbei fiel schon macroscopisch eine grosse Verschiedenheit in dem Aussehen der Schnittfäche auf. Während dicht hinter dem Eintritt des Gefässes nur eine kleine dreieckige Figur, mit der Basis der Scheide zagewand, von etwas weisserer Färbung und glatterer Oberfläche sich deutlich und scharf begrenzt gegen die mehr gekörnte Fläche der übrigen Nervensubstanz absetzt, nimmt dieselbe, je weiter die Querschnitte dem Bulbus sich nähern, immer mehr an Grösse zil. Die Spitze des Dreiecks liegt in einer Entfernung von $8 \mathrm{Mm}$. vom Bulbus (wo der Querschnitt des Nerven eine Ausdehnung von $2^{1 / 2} \mathrm{Mm}$. in dem einen und $2^{3 / 4} \mathrm{Mm}$. in dem anderen Durchmesser zeigt) noch ziemlich nahe der inneren Nervenscheide; auf einem Schnitte, der etwa $4 \mathrm{Mm}$. vom Bulbus entfernt, ist sie schon bis über das Centrum hinausgerückt, indem sich gleichzeitig auch die Basis verbreitert. Die glatt und blass aussehende Fläche nimmt hier einen Sector ein, der etwa ein Viertel der ganzen Kreisfläche repräsentirt. Sie ist der optische Ausdruck einer zunehmenden Atrophie des Nervengewebes. Je mehr man sich dem Bulbus nähert, um so grösser wird dieselbe; gleichzeitig verliert der ganze Nerv an Umfang.

Die microscopische Untersuchnng der Querschnitte ergiebt, dass die Arterie bei ihrem Durchtritt durch die Scheide und in ihrem allerfruhesten Verlauf im Opticus selbst noch ein freies Lumen hat. Hier schon giebt sie einen ziemlich erheblichen Ast $a b$, der von jetzt an parallel mit ihr nach dem bulbus hin verläuft und auf den Querschnitten neben ihr deutlich hervortritt: auch dessen Lumen ist im ersten Verlauf frei. Bald aber finden sich beide Gefässe verstopft. Ein Schnitt, an 
einer Stelle, wo schon die Vene hinzugetreten, zeigte folgendes Bild (siehe Figur 1). Die Bindegewebsscheide der Gefässe tritt deutlich hervor und erscheint verbreitert. Neben ihr liegen Nervenbündel, deren Querdurchschnitt abnorm klein ist. Durch die breite Bindegewebsscheide und die atrophischen Nervenbündel, welche nach einer Richtung hin bis zur Scheide auftreten, wird die obcu beschriebene, schon macroscopisch sichtbare, Dreieckähnliche Figur gebildet. Die Atrophie der Nervenfasern wird auch durch das Ausbleiben der Goldchlorid-Reaction erwiesen, die in den mehr breiteren Bündeln des übrigen Sehnerven hier noch meist deutlich hervortritt. Die Arteria centr. retinae erscheint etwas verengt. Díc Contouren des Lumens werden durch glänzende Wellenlinien der Intima gebildet. Im Innern des Gefâsses, dasselbe vollständig ausfüllend; liegt eine, grösstentheils hyaline, gelbliche Masse, die durch Querlinien in verschiedene unregelmässige Abtheilungen zerfälit (cf. Fig. 2). Während die grössere Partie dieser Substanz vollkommen homogen und zellenfrei erscheint, finden sich besonders an einer Seite, in der Nähe der Wandung, rundliche, verschieden gezeichnete und verschieden grosse Figuren. Einzelno derselben haben vollkommen das Aussehen weisser Blutkörperchen; andere sind grösser, zeigen ebenfalls einen gekörnten Inhalt und mehrere Kerne. Schliesslich tritt noch eine Formation hervor (Figur 2c), die eine circuläre gestreifte Umbüllung hat, welche sich scharf gegen ein Lumen absetzt. In diesem liegen mehrere zellige Gebilde, die etwa das Aussehen rother Blutkörperchen haben. Beim Verschiebèn des Microscoprohres lässt sich diese Bildung durch dic ganze Dicke des Schnittes verfolgen: es handelt sich demnach um einen cylindrischen Schlauch, und die Vermuthung erscheint nicht ungerechtfertigt, dass wir es hier mit einem Blut- 
gefäss zu thun haben, das sich in dem Thrombus gebildet hat.

Die Wände der Arteria centralis zeigen nichts abnormes, weder Zellen-Einlagerungen noch Verkalkungen. - Neben ihr befindet sich der schon oben erwăhnte Seitenast (Fig. 1b). Auch dieser lässt ähnliche Schlängelung der Intima erkennen und ist ebenfalls durch eine hyaline Masse obturirt. Weiterhin finden sich noch Querschnitte verschiedener kleinerer Gefässe (Fig. 1d, e, b), bei denen zum Theil ein offenes Lumen, aucli bei stärkerer Vergrösserung Epithel an der Intima, zu bemerken ist. Dis Mehrzahl derselben sind als Arterien anzusprechen. Die Vene ist collabirt, zum Theil erfüllt mit Blutgerinnsel (Figur 1v).

In den Scheidewänden zwischen den Nervenbündeln liegen ebenfalls, hier und da zerstreut, Querschnitte von Blutgefüssen, von denen besonders die mit $f$ und $g$ in Figur 1 bezeichneten, hervortreten.

Der dem Bulbus nächstgelegene Theil des sehr verdünnten Sehnerven wird durch Längsschnitte getheilt. Die sonst unterhalb der Lamina cribrosa erkennbare bündelförmige Anordnung und dunklere Färbung der Nervenfasern ist verschwunden: es findet sich hier dasselbe helle, längsstreifige Gewebe, wie in der Papilla optica. Die Lamina cribrosa, nicht immer deutlich zu verfolgen, zeigt eine leichte Concavität nach dem Bulbus zu. Das Scleralgewebe grenzt sich nicht so scharf wie sonst gegen den Nerven ab, sondern geht an der Durchtrittsstelle verschwommen und alimälig in denselben über.

Die Papille selbst besteht aus streifigem Bindegewebe, erscheint etwas vertieft und verkleinert. An einzelnen Stellen setzt sich das Gewebe obue jede Abgrenzung weiter fort und bildet eine Membran, die als Rest der Retina und Chorioidea sich dem Opticus anschliesst. Hier findet man dann auch bisweilen schwarzes Pigment noch in das Gebiet der Papille hineingeschwemmt. Gefässe 
sind sehr sparsam. Auf einem Schnitt siebt man den Querschnitt einer grösseren Arterie, die von einer hyalinen, gelblichen, in verschiedene Figuren zerfallenden Masse, verstopft ist; auf einem anderen kömmt von der Sclera her -.- quer durch die Papille - ein ziemlich dicker Gefässast, der zum Theil mit einem schwarzen Gerinnsel erfüllt ist. Mitten aus der Papille, nach dem Glaskörper zu, geht ein durchsichtiger, structurloser, aber bräunlich gefärbter Fetzen der Membrana hyaloidea.

In den lockigen Bindegewebsbündeln, welche die Sehnerven-Scheide dicht an der Sclera bilden, liegen ziemlich grosse, runde und eirunde Körper, von meist concentrisch geschichtetem Bau, die bröckliche und beim Schnitt knirschende Massen bilden und sich als Psammome (wie im Hirnsande) legitimiren (siehe Figur 3). Die grösseren haben einen körnigen, dunkleren Kern, um den die concentrischen Schicbten, welche an der Peripherie wiederum von einem etwas breiteren Rande umsäumt werden, sich anlagern; bei den kleineren fehlt die Schichtung. Bei Zusatz von Salzsäure treten kleine Bläschen auf ihnen hervor. Ihre Grösse wechselt im Durchmesser von $0,03-0,15 \mathrm{Mm}$. Durch diese Grösse, sowie das Fehlen der Jodreaction unterscheiden sie sich von den Amyloidkörpern. Sie stimmen, wie mir scheint, mit den Gebilden überein, die Leber*) ebenfalls in der Scheide eines atrophischen Sebnerven gefunden und als Bindegewebs-Bildungen den geschichteten Formationen gleichgestellt, die H. Müller im Ciliarmuskel gelegentlich einmal beobachtet hat.

Nach der Halbirung des Bulbus im Aequator zeigt dic Netzhaut und Chorioilea im hinteren Augenabschnitt schon macroscopisch ein, an den einzelnen Partien verschiedenes Verhalten. Von der Papille aus erstrecken

*) Arch. f. Ophth., XIX. 1, S. 199. 
sich hellgelbliche Figuren, mit unregelmässigen Fortsätzen, sowohl in der Richtung der Macula lutea hin, die selbst nicht mehr zu erkennen ist, als auch nach innen zu. Der grössere Theil des Augengrundes hat eine mekr grünliche Färbung angenommen. Bei den Versuchen, die Retina von der Chorioidea zu lösen, ergiebt sich, dass an den helleren Partien eine innige Verklebung stattgefunden; bisweilen sind beide Membranen in eine einzige umgewandelt, die kaum die Dicke hat, welche sonst einer normalen Netzhaut zukommt. Microscopische Durchschnitte zeigen hier, dass die specifischen Netzhautelemente geschwunden sind; an ibre Stelle ist ein streifiges Bindegewebe getreten; ebenso fehlt meist das Pigment-Epithel. Im Stratum der Chorioidea finden sich noch hier und da Pigmentzellen, die zu kleinen Haufen zusammen getreten sind.

An anderen Partien ist die Netzhaut besser erbalten; die radiären Stützfasern sind stark verbreitert, ofter fehlt die granulirte und innere Körnerschicht. Die Wandungen der Arterien sind verdickt, das Lumen ist oft nur noch durch eine feine Linie repräsentirt. $\mathrm{Zu}$ weilen sieht man in einer mittelgrossen Arterie, dass ein Blutkörperchen dasselbe vollkommen ausfültt, während dicht daneben Capillaren eine Masse derselben enthalten. Die einzelnen Schichten der Gefässwand sind nicht deutlich von einauder zu scheiden; das Epithel meist gut erhalten. Die Venen sind häufig stark mit Blut gefüllt.

Um grössere Gefässstrecken zu übersehen, wurden besser erhaltene Netzhautstücke in dünne Kalilösung gelegt. Es fand sich hierbei, dass die Lösung im Ganzen nicht so lichtend wirkte, als auf Partien gesunder Netzhaut - von anderen Augen -, die gleichzeitig mit hineingelegt wurden. Bei dieser Durcbsicht sah ich einmal in ienem Gefässe, das seiner Grösse nach einer 
kleinen Arterie entsprach, einen eiförmigen Embolus, gerade an der Stelle sitzend, wo das Gefäss sich in zwei ungleich grosse Aeste theilte. Drei Viertel desselben lagen in dem grösseren Ast, während ein Viertel etwa noch in den Hauptstamm hineinragte.

In der Chorioidea, an Stellen, wo sie besser erhalten war, zeigte sich die Chorio-Capillaris oft stark hyperämisch; auch fanden sich hier und da in ihr Blut-Extravasate. An den Gefässwandungen wurden keine $\mathrm{Ab}$ normitäten bemerkt.

Alle pathologischen Veränderungen in der Chorioidea und Retina nahmen $a b$ und wurden geringer nach dem Aequator zu.

Der Glaskörper war zähe und haftete zum Theil ausserordentlich fest der Netzhaut an. In Wasser gethan, erscheint er in grosser Anslehnung von einer zarten, weissliche Falten bildenden Membran umgeben, die sich ablösen lässt und unter dem Microscop durchsichtig und structurlos ist. Dicht unter ihr - dem Glaskörper zu - liegen an einzelnen Stellen gelblich-röthliche, unregelmässige Brocken, die aus einer Masse rother Blutkörperchen bestehen. Die Substanz des Glaskörpers ist nicht vollkommen homogen, sondern gewährt ein feingekörntes Aussehen, als wenn ihr unendlich viele kleine Luftbläschen imprägnirt wären. Selbst starke Vergrösserungen lassen in diesen kleinen Kreisen keine Differenzirungen erkennen.

Der ophthalmoscopische Befund weicht nicht wesent lich vou dem $a b$, was andere Beobachter gesehen. Bemerkenswerth bleibt die frühzeitig anftretende Trübung des Netzhautgewebes zwischen Sehnerv und Macula latea, welche schon innerhalb der ersten 20 stmiden beobachtet wurde: ähnlich im Uebrigen wie in dem lialle von M egi. 
höfer*), der sie 16 Stunden uach der Erblindung constatirte. Ebenfalls atn ersten oder in den ersten Tagen sahen sie Liebreich**), Blessig***), Just Fano + ), Steffantti), Knapp ${ }^{*} \dagger$ ) (2. und 3. Fall) and Schirmer**) auftreten teh glaube hieran erimnern 2u müssen, da Magnus***t) nenerdings versucht hat, in dem frühzeitigen Erscheinen dieser Trübung ein diagnostisches Moment gerade für Blutungen in den Sehnerven - im Gegensatz zu Embolien der Arter. central. retin. - aufzustellen. Er schreibt (1. c. p. 50): „Findet sich hierrach bei einer fulminanten Erblindung schon in den nächsten Stunden oder Tagen nach dern Auftreten derselben eine circummaculare Tribung and Röthung der Macula selbst, so können wir mit Bestimmtheit annehmen, dass eine Embolie der Central-Arterie dieselbe allein nicht hervorgerufen haben kann, sondern dass ein anderer Factor in Wirksamkeit gewesen sein muss. Da wir nun aber sowohl auf dem Wege des Experiments als der klinischen Erfahrung zu der Erkenntniss gelangt sind, dass die Netzhaut gegen jede Verletzung des Sehnervenstammes in schnellster und promptester Weise mit einer granen Trübung antwortet, so werden wir uns wohl keines Trugschlusses schuldig machen, wenn wir für die plötzlich mit Blindheit aultretende Amblyopie, welche schon bald in den ersten Stunden ihres Bestehens von einer grauweissen Trübung

*) Ueber Embolie der Art. centr. retinae, Inaugural-Dissertation. Königsberg 1873.

**) Annales d'oculist. T. XLVII, p. 124.

***) Arch, f. Ophth. VIII, 1, p. 216 .

†) Kl. Monatsblätter f. Augenheilk. 1863, 1. 265.

†) Gaz. des hôpitaux 1864 p. 482 . - Annales d'oculist. T. 52.

tit) Arch. f. Ophth. XI, 1.

* f) Arch. f. Ophth. XIV, 1, p. 210-220.

*** K) Kl. Monatsblätter f. Augenheilk. 1868, p. 38.

****at) Die Sehnervenblutungen 1874, p. 46 u. folg. 
der Netzhaut gefolgt ist, eine traumatische Beleidigung als genetisches Moment annehmen".

Mein Fall lehrt nun, dass man sich in dieser Weise allerdings eines Trugschlusses schuldig machen kann: es war eben weder ein Trauma noch eine Sehnerven. Blutung, sondern eine einfacbe Verstopfung der Arteria central. vorhanden.

Leider hat die anatomische Untersuchung mir keine Auskunft über die Ursache des rothen Fleckens oder Streifens in der Gegend der Macula lutea, die noch Gegenstand der Discussion ist, gehen können, da die folgenden Entzündungen das Bild verwischten. Nach dem ophthalmoscopischen Befunde zu schliessen, möchte ich indessen annehmen, dass es sich eher um eine Blutung als um eine Contrast-Wirkang - wie v. Graefe*) es will - handelte. Dafür spricht einmal das Aussehen, welches strichförmig und durchaus nicht der Form dex Macula lutea gleich erschien, und weiter der Sitz. Der rothe Streifen befand sich an einem Rande, nicht in der Mitte der Macula, wie man es doch, wenn es sich um eine Contrast-Wirkung bandelte, hätte erwarten müssen, da wegen der Dünnheit der Retina-Schichten das Chorioidealpigment hier am stärksten durchschimmern kanu. Schon am nächsten Tage war der dunkle Strich bei zunehmender Trübung verschwunden. Da die Resorption des Blutes nicht so schnell erfolgt sein kann, so scheint eine weiter fortschreitende Infiltration der vorderen Netzhautschichten ihn verdeckt zu haben. Die Blutung hätte demnach ir den tieferen Schichten der Retina oder auch in der Chorioidea ihren Sitz gehabt.

Ganz eigenartig ist der beschriebene Krankheitsfiall nach der Richtung, dass nach der Embolio der Arter. centralis retinae sich anch noch das klinische Bild um-

*) Arch. f. Ophth. V, 1, p. 150. 
bolischer Verstopfung von Ciliar-Gefässen unter der Form einer Irido-Chorioiditis entwickelte. Bei der Besprechung der Steffan'schen Auffassung der Embolie der Arteria centralis wird dieser Verlauf noch Berücksichtigung finden.

Der anatomische Befund in der Sehnerven-Arterie stimmt ungefähr mit dem überein, den Sichel fils gehabt und abgebildet hat. Auch bei ihm ist die Intima gefaltet, doch erscheinen in der Verstopfungsmasse die Rundzellen zahlreicher als bei mir, wo der grösste Theil aus einer homogenen, durehscheinenden Substanz bestand. Finzelne Schnitte Sichel's (cf. I. c. Fig. 4) zeigen allerdings auch ein ähnliches Bild, so viel aus den eben nicht sehr hervorragenden Zeichnungen abzunehmen ist.

In meinem Präparat begleitete, wie erwähnt, ein parallel verlaufender Seitenast die Hauntarterie fast durch die ganze Länge des Nerven. Derselbe ist im Anfang noch durchgängig, später aber ebenfalls obturirt. Andere kleinere Gefässe, mit zum Theil freiem Lumen, sind ausserdem noch in dem centralen Bindegewebsringe vorhanden. Auch in den Scheidewänden, welche dio Nervenbündel umgeben, besonders dort, wo letztere noch normal sind; finden sich ungewöhnlich zahlreiche Gefässe. Es scheint sich demnach hier und da ein Collateral-Kreislauf gebildet zu haben, sei es von dem noch freien Anfangstheil der Arter. centralis oder ihrem Nebenaste ausgehend, oder auch von den Gefässen der Sehnervenscheide.

Dass die verstopfende Masse in ihrem wesentlichen Theil wirklich als Embolus, nicht als Thrombus aufzufassen ist, geht sowohl aus dem Krankheitsverlauf als auch aus dem anatomischen Befunde hervor. Die Erblindung war ganz plötzlich erfolgt; zuerst war die Netzhautarterie, kurz darnach waren Ciliaräste undurchgängig geworden. Der organische Herzfehter giebt uns ferner den Ausgangspunkt embolischer Gerinnungen. In Gehirn selbst zeigt die Section Reste eines embolischen Heerdes. 
Aber auch an der Verstopfungsmasse sehen wir nichts von den Veränderungen, die an einem Thrombus bei längerem Bestehen - und anser Patient lebte noch $3 / 4$ Jahr - aufzutreten ptlegen. Es fehlt sowohl eine Umwandlung in einen gefässreichen Bindegewebspfropf, noch treten die Erscheinungen, welche sich mit einer Erweichung des Thrombus verbinden, hervor. Die reichlichere Zellenbildung, die sich in einem Theile der Verstopfungsmasse in einer Reihe von Präparaten findet, sowie auch die ebenfalls daselbst vorhandene und für einen Gefässquerschnitt angesprocheue Figur scheint darauf hinzuweisen, dass der Embolus hier nicht ganz das Lumen der Arterie ausgefüllt hat und die vollständige Verstopfung hier in der That durch ein sich bildendes Blutgerinnsel, das sich später in einen vascularisirten Thrombus umwandelte, erfolgt ist.

Es fehlen ferner auch jedwede ortlichen Ursachen, welche eine primäre Thrombosirung veranlassen könnten. Die Arterienwand zeigt keine pathologischen Verände. rungen; ebensowenig ist in dem umliegenden Gewebe eine Schwellung oder Exsudation bemerklich, die eine Compression und Verschluss des Arterienrohres hätte béwirken können - selbst wenn man diese Möglichkeit zugeben wollte. v. Graefe*) hat bekanntlich die Ansicht ausgesprochen, dass eine retrobulbäre Neuritis durch Einwirkung auf die Aussenfläche der Arterie eine Thrombose in ihr bewirken könne und dass eine Reihe von Fälen, die als Embolie beschrieben, so ihre Deutung finden. Was ich nach dieser Richtung hin gesehen, spricht nicht dafür: ich halte die Resistenz der Wand der Arter. centralis für viel zu mächtig, als dass entzündliche Schwellungen eine zum Verschluss führende Compression ausüben könnten. Man beobachte nur die

*) Arch. f. Ophthaim. XII, 2, p. 143 
colossale Schwellung des Gewebes, die Exsudation und Zellen-Einlagerungen in dea Fälen von Stauungs-Papille - und dennoch bieibt tine Thrombose der Stammarterie aus. Weitere klinische Bedenken, die gegen v. Graefe's Anschaung sprechen, sind schon von anderer Seite hervorgehoben.

Schliesslich ist hier noch der deutlich abgegrenzte Embolus in einem kleineren Arteriengefäss der Netzhaut $z u$ erwähnen.

Die eigenthümliche Gestalt und Figur, welche schon macroscopisch die atrophischen Partien des Sehnerven boten und die zu dem Verlaufe der Centralarterie in Beziehung stand, findet wohl so ihre Erklärung, dass zuerst, die Partien der Nervensubstanz zu Gronde gingen, welche den obturirten Gefässen am nächsten lagen und vorzugsweise auf die Ernähung aus den verstopften Aesten angewiesen waren.

Was die Gefässe der Netzhaut betrifft, so ist erwähnt, dass die Peripherie damit ziemlich reichlich versehen war und dass die Arterien daselbst am meisten Bht führten. Wir dürfen in unserem Falle dies wohl auf eine Gefässverbindung mit der Chorioidea zurückführen, wozu die vorangegangene Entzündung, die Verklebungen und Verwachsungen beider Membranen bewirkte, ausreichende Veranlassung gegeben hat.

Nicht so leicht aber sind bekanntlich die Fälle von Embolie zu erklären, bei denen ohne Entzündung und davon abhängige Verklebung später sich wieder eine mehr oder weniger ausgedehnte Füllung der Arterien eingestellt hat. Und diese Fälle sind nicht so selten: K na p p(l.e.), F a no

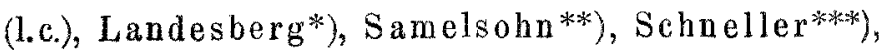

*) Arch. f. Ophthalm. XV, 1, p. 214.

**) Arch. f. Augen- u. Ohrenheilkunde 1II, I, p. 130.

$* * *$ ) Arch. f. Ophthalm. VIII, 1, p. 271. 
Blessig, Hock*) haben derartige Beobachtungen verzeichnet. Bei den letzten drei Autoren wird direct die Papille resp. die Gegend derselben als Ausgangspunkt der erneaten Blatcirculation angegeben. Man recurrirte bis jetat zar Erklärung dieser Erscheinung meist auf Gefässäste, welche aus hinteren Ciliararterien stammend, durch Vermittlung des Zinn'schen Scleralkranzes normaler Weise Blut in die Papille führen.

Steffan ging, hierauf basirend, noch weiter. Er hielt diese Communication für so ansreichend, dass ,eine Verstopfung der Arteria centralis retinae allein höchstens eine 24stündige Kreislaufstörung in der Retina hervor bringen könne". Nach ihm sind daher alle anderweitigen Beobachtungen als Embolien der Arter. centralis retinae sammt den hinteren kurzen Ciliararterien oder mit anderen Worten als Embolien der Arteria ophthalmica aufzufassen. v. Wecker bält noch nonerdings in seinem Traité des maladies du fond de l'oeil (1870) diese Theorie aufrecht.

Es ist hiergegen schon a priori eingewandt worden, dass bei einer derartigen Unterbrechung in den Ciliarästen auch ausgesprochene Verändernngen in dem Aussehen der Chorioidea, entzündliche Erscheinungen hier, in der Iris und dein Glaskörper auftreten müssten. Die oben von mir mitgetheilte Beobachtung hat jetzt den factischen Beleg dafür geliefert.

Aber auch anatomische Bedenken, auf welche neuerdings Leber**) wiederholt hingewiesen, stehen der Auffassung Steffan's entgegen. Die in Rede stehenten Aeste des Scleralkranzes sind nämlich sehr fein und

*) Wiener med. Presse 1869, Nr. 44.

***) Arch. f Ophth. XVIII, 2, p. 32. - Nagel's Jahresbericht $x, p .314 \mathrm{a}, 343$. 
lösen sich schnell in Capillaren auf. Nach Leber's Ansicht sind dieselben überhaupt nicht stark und zahlreich genug, um einen hinreichenden collateralen Zufluss zu ermöglichen.

Wenn demnach in dieser Weise der Steffan'schen Theorie der Boden unter den Füssen fortgezogen ist, so ist uns aber auch gleichzeitig uberhaupt die Erklärung für die Wiederherstellung ergiebiger Blutcirculation genommen. Es drängt sich daher die Vermuthung auf, dass noch anderweitige Verbindungen des retinalen resp. papillaren Gefässsystems existiren und wenn $M$ a u thner*) hierbei sein Augenmerk besonders auf die Aderhaut richtet und neue Untersuchungen über den Gefässverlauf in Netz- und Aderhaut verlangt, so dürfte moin Fall dazu angethan sein, auch den Gefässen im Sehnerven eine gewisse Aufmerksamkeit zuzuwenden.

Bei meinen Präparaten war, wie erwähnt, in einer grossen Reihe von Querschnitten neben der eigentichen Arter. centralis retinae noch eine zweite, ziemlich grosse Arterie - ebenfalls obturirt - in dem Bindegewebsstrange nachweisbar. Um eine Anschaung von dem Grössenverhältniss zwischen beiden zu geben, habe ich in einem Präparat das Lumen gemessen. Da dies bei den Wellenlinien der Intima seine Schwierigkeit hat, so beanspruchen die gefundenen Zahlen nur eine relative Richtigkeit. Die Arter. central. maass in einer Richtung ihres Kalibers. 0,15 Mm., in der anderen 0,08; die kleinere Arterie hingegen 0,06 resp. 0,03 . Wir haben es demnach bier mit einem ziemlich erheblichen Gefässast zu thun, der bis in die Nähe des Bulbus reicht.

Dieser Arterienast ist nun auch sonst häufiger vor-

*) Medicinische Jahrbücher, herausgegeben von der K. K. Gesellschaft der Aerzte. 1873 , pag, 195. 
handen. Schwalbe*) schreibt so: „Nur selten sind an Querschnitten dieser Gegend die Arteria und Vena centralis die einzigen Blutgefässe des Bindegewebsstranges. Vielmehr bemerkt man an fast allen Querschnitten dieses Sehnerven-Abschnittes nach aussen von der grösseren Arteria centralis noch den Querschnitt eines kleineren arteriellen Gefässes ..... Längsschnitte durch den centralen Bindegewebsstrang ergänzen diese Bilder und zeigen nun, wie dicht an der Stelle, wo die Centralartérie die Opticusaxe erreicht hat und in derselben nach dem Augapfel zu sich wendet, eine etwa 0,07 Mm. dicke Arterie von ihr ihren Ursprung nimmt, um, sich allmälig verjüngend, dicht neben der Centralarterie und parallel derselben bis nahe an die Sclera weiter zu verlaufen. Dabei giebt sie nach aussen zwischen die Opticusfaserbündel zahlreiche Aestchen ab, die mit den Capillaren dieser Gegend anastomosiren".

Es dürfte nun sehr annehmbar erscheinen, dass in Fällen einer Verstopfung der Arter. centralis, die erst hinter dem Abgange des Seitenastes ihren Sitz hat, dieser letzere einen collateralen Kreislauf und eine ausreichende Speisung der papillaren Gefässe einleitet: un so leichter als nun die ganze Blutmenge, die überhaupt in den Sehnerven eindringt, sich ihm zuwenden muss. Und nach dieser Richtung ist er im erheblichem Vortheil gegen die hinteren Ciliaräste, wenn man diesen eine Rolle bei der späteren Wiederherstellung der papillaren Blut-Circulation zuschreiben wollte. Bei dem allerdings variablen Ursprunge derselben, der aber meist sich ziemlich entfernt von dem der Art. central. retin. findet, ist jedenfalls auf eine bei Verstopfung der Art. centralis eintretende, erhebliche Vermehrung des Blutzuflusses oder

*) Graefe-Saemisch, Handbuch der gesammten Angenheilkunde I, 1, p. 346 .

จ. Graefe's Archiv für Ophthalmologie, XX. 2. 


\section{6}

Steigerung des Blutdruckes in ihnen nicht zu rechnen. Hiermit aber fällt eine der Ursachen zur schnelleren Ausbildung eines collateralen Kreislaufes fort, die dem Seitenaste der Arter. centralís zu Gute kömmt.

Meiner Auffassung nach ist das Zustandekommen einer erneuten Blutcirculation in einer Reihe der als Embolie der Art. central. retinae beschriebenen Fälle demnach in der Weise zu deuten, dass der Embolus seinen Sitz jenseits der Abgabe des Seitenastes hatte und dass dieser letztere dann den collateralen Kreislauf einleitete. In anderen Fällen, wo die Gefässe verödet blieben, mag der Embolus vor dem Abgange des Seitenastes das Lumen verstopft haben oder auch, es sind, wie in meinem Falle, embolische Massen in beide Gefässe hineingeschwemmt. Noch bleibt die weitere Möglichkeit, dass dieser Zweig überhaupt fehlt. - Mit der Aufstellnng dieser Erklärung will ich keineswegs behaupten, dass nicht auch noch in anderer Weise ausreichende collaterale Kreisläufe, sei es von der Chorioidea oder vom Corpus ciliare her, zu Stande kommen könnten. Manche Fälle sprechen sogar dafür: doch ermangeln bis jetzt noch die anatomischen Belege. 


\section{Erklärung der Abbildungen.}

Figur 1. Querschnitt durch den Sehnerven. 60 mal vergrössert.

a Arteria centr, retin. obturirt.

b Hauptast derselben, ebenfalls obturirt.

$c, d, e, f, g$ Kleinere Gefäss - Querschnitte. An den Arterien c und e, deren Lumen erhalten ist, sieht man bei stärkerer Vergrösserung das Epithel.

$\checkmark$ Vena centr. retinae, zum Theil Blutgerinnsel enthaltend.

Figur 2. Querschnitt durch die obturirte Arter. centralis retinae. 500 mal vergrössert.

a Hyaliner Embolus, in einzelne Abschnitte zerfallend.

b Peripherer Theil mit Zellen durchsetzt.

c Querschnitt eines Gefăsses.

i Wellenförmig gefaltete und stark glänzende Intima.

Figur 3. Psammomartige Bildung aus der Sehnervenscheide $300 \mathrm{mal}$ vergrössert.

a Centraler Kern, dunkler und körnig.

b Concentrische Schichten.

c Aeusserste, mehr gleichmässige und breitere Umbüllung. 

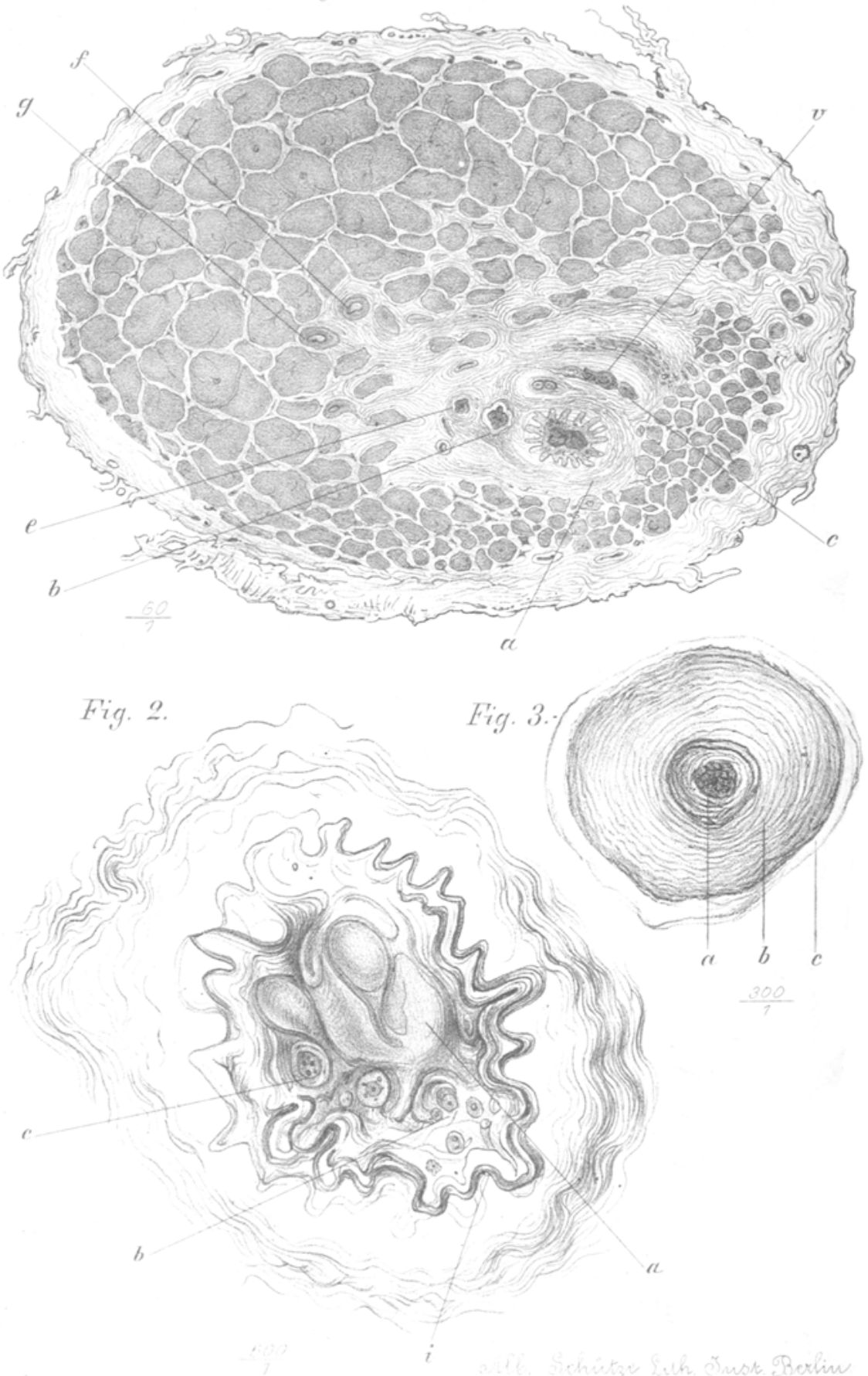